\title{
FI LYR: A CANDIDATE BINARY SYSTEM CONSISTING OF CARBON-RICH AND OXYGEN-RICH COMPANIONS
}

\author{
J.J. WANG, J.Y. HU and X. ZHOU \\ Beijing Observatory, Academia Sinica, Beijing, 100080, China
}

FI Lyr=IRAS18401+2854 is listed in the GCVS as type of SRb, the spectral type is M. In the IRAS LRS Catalog it is classified as 41 , namely having the $\mathrm{SiC}$ feature at $11.3 \mu \mathrm{m}$ from a carbonrich dust shell. We have obtained optical and near infrared spectra in the same period, but they show different chemical natures. We suggest that FI Lyr is a good candidate of a binary system consisting of carbon-rich and oxygen-rich companions.

The near infrared spectrum was obtained on April 2, 1992 using the $1.26 \mathrm{~m}$ telescope at Beijing Observatory equipped with CVF spectrometer and InSb detector. The coverage of near infrared spectrum is from $2.9-4.0 \mu \mathrm{m}$, in which clearly shows the feature of $\mathrm{C}_{2} \mathrm{H}_{2}$ and $\mathrm{HCN}$ at $3.1 \mu \mathrm{m}$ commonly observed in the carbon stars. The optical spectrum was obtained on March 10, 1992 using the $1.93 \mathrm{~m}$ telescope equipped CARELEC at Haute-Provence Observatory. A linear CCD was used as the detector. It is a typical late M-type spectrum. We found in the IRAS LRS spectrum of FI Lyr an emission feature from 9 to $12 \mu \mathrm{m}$ and centred at $10.7 \mu \mathrm{m}$. It is longer than silicate feature at $9.7 \mu \mathrm{m}$ of oxygen-rich objects and shorter than $\mathrm{SiC}$ feature at $11.3 \mu \mathrm{m}$ existed in carbon stars. Additionally there is a hump around $18 \mu \mathrm{m}$, resemble with silicate emission from oxygen-rich object.

Skinner et al.(1990) observed the near infrared spectra of a group of M-type stars with SiC feature and carbon stars with silicate feature and found that the near infrared chemical nature is always identical with the optical. But this is not true in the case of FI Lyr. We consider FI Lyr to be a binary system consisting an M-type giant star and a carbon star.

Skinner,C.J., et al., 1990,MNRAS,243,78. 